\title{
Problèmes hémodynamiques des prothèses cardiaques
}

\section{Haemodynamic problems associated with cardiac protheses}

\author{
Louis Vadot \\ Ingénieur-Docteur \\ Docteur en Biologie Humaine, Hôpital Cardiologique de Lyon
}

\section{Rappel de notions fondamentales}

Le système circulatoire peut être représenté très schématiquement par une boucle le long de laquelle figurent quelques singularités, les unes génératrices, les autres consommatrices d'énergie. L'apport d'énergie par les pompes ventriculaires droite et gauche est très localisé, la consommation par contre est plus ou moins régulièrement répartie le long des deux circuits pulmonaire et systémique. Tout cet ensemble monté en série dans une même boucle complètement fermée doit s'accommoder d'un débit identique dans chacun de ses quatre constituants fondamentaux. C'est tout au moins ce qui doit se passer en moyenne pour un régime établi, mais l'examen attentif révèle des écarts temporaires par rapport à cette situation idéale soit sous l'effet de variations ventriculaires, soit sous l'effet de modifications des caractéristiques des circuits vasculaires.

Il est nécessaire de rappeler très brièvement l'essentiel de ces caractéristiques. Les unes, dynamiques, gouvernent l'ensemble résistance, débit, pression, les autres, statiques, commandent la répartition des volumes.

Les deux groupes de valeurs sont liés par l'intermédiaire de l'élasticité d'une part, de la résistance, ellemême sous la dépendance des diamètres, d'autre part. Pour un élément du circuit vasculaire la chute de pression est donné par la loi de Poiseuille

$$
\Delta p=A \cdot Q \cdot \frac{1}{D^{4}}=Q \cdot R
$$

$A$ étant une constante, $Q$ le débit, $D$ le diametre, et $R$ la résistance.

Quant à la variation de volume elle est de la forme :

$$
\Delta V=C . \Delta p
$$

$C$ étant une caractéristique, dite compliance, définissant l'élasticité du système. Or $C$ varie avec l'état de disten- sion des tissus, par suite de leur structure ; il varie également avec la vaso-tonicité sous la dépendance des régulations.

Pression, résistance, volume et compliance varient le long du circuit. Les points essentiels sont : la forte localisation de la résistance (surtout systématique) dans la zone précapillaire, le grand volume et la forte compliance des territoires veineux. Du point de vue des évolutions régulatrices, on notera les variations des résistances précapillaires, les variations des volumes veineux. A cela, on ajoutera enfin la variation du volume sanguin total sous l'influence des pertes liquidiennes et des apports alimentaires ou par perfusion.

Chacun des deux cœurs comporte une cavité assez passive, l'oreillette, et une cavité dynamique, périodiquement contractée, le ventricule. L'oreillette (dont la contraction est négligée sur les prothèses) n'est qu'un prolongement du système veineux, à la compliance duquel elle participe par son élasticité propre.

En période systolique, le ventricule par sa contraction fournit débit et énergie. En période diastolique par contre, son comportement est passif, il se dilate et se

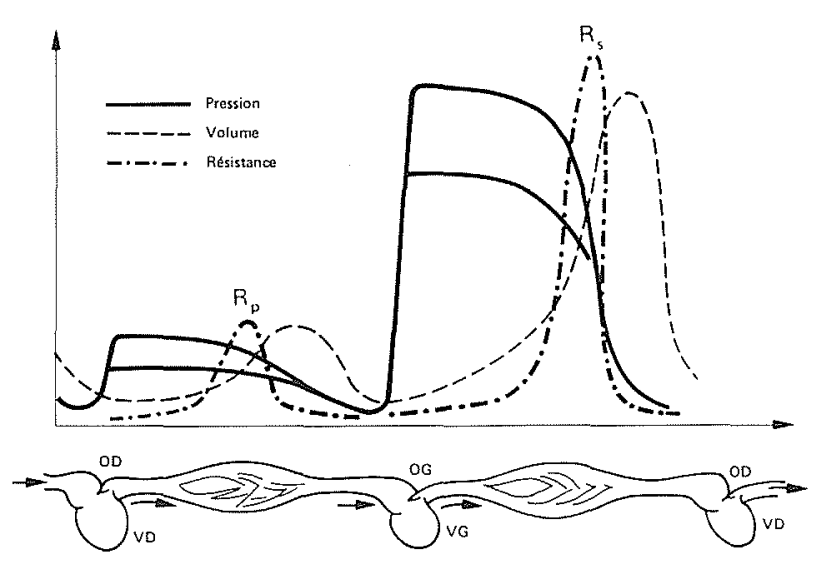

Figure 1 - Evolution des caractéristiques hémodynamiques. 
remplit sous l'effet de la pression veineuse (ou auriculaire) restante. Sa compliance diastolique joue alors un double rôle d'une grande importance.

D'elle dépend le remplissage, donc le volume systolique et le débit. Elle commande en outre, par la dilatation télédiastolique atteinte, les possibilités de contraction ultérieure, donc la pression. Finalement cette compliance ventriculaire diastolique, bien négligée dans les traités classiques, est à la base de l'énergétique cardiaque. On la retrouvera comme un des éléments fondamentaux de la construction des prothèses.

\section{Prothèse cardiaque totale}

D'un point de vue très général une prothèse cardiaque totale est une vulgaire pompe à laquelle on demande débit et pression. On notera tout de suite que si la prothèse impose le débit, la pression, quant à elle, est imposée par le réseau vasculaire, en sorte que le fonctionnement circulatoire n'est que partiellement sous la dépendance artificielle de la commande. On ne peut donc en aucun cas ignorer le comportement du réseau vasculaire et de ses régulations.

On peut penser, au premier abord, que la forme sous laquelle est fourni le débit est sans importance. En particulier on a essayé le système à débit continu non pulsé qui se prête bien à l'emploi d'une rotation rapide. Une pompe centrifuge a ainsi été proposée, toutefois la nécessité physiologique de la pulsation a conduit à faire varier périodiquement la vitesse de rotation pour obtenir des ondes artérielles. Dans ces conditions, la prothèse à pulsation par ventricule élastique du genre imaginé par Kolff est presque exclusivement retenue. Notons toutefois que le fonctionnement en phase des deux cœurs n'est pas une nécessité. Une double prothèse en opposition de phase droite et gauche a d'ailleurs été expérimentée.

L'emploi d'une seule prothèse, avec un seul mécanisme d'activation a également été étudié dans un but de simplification du dispositif de commande. La différence des niveaux de pression droite et gauche conduit toutefois à l'emploi d'un étranglement réducteur, soit sur le circuit sanguin (à la manière d'une sténose de l'artère pulmonaire), soit sur le circuit de commande.

On a également essayé un montage à commande unique avec une communication interventriculaire combinée au rétrécissement pulmonaire. C'est une curiosité sans grand intérêt.

Bien entendu toute prothèse cardiaque doit satisfaire à un certain nombre de conditions :

\section{1. en ce qui concerne la forme:}

- elle doit s'adapter à l'espace médiastinal sans gêner les mouvements pulmonaires,

- elle doit se brancher facilement sur les gros vaisseaux ;

\section{2. en ce qui concerne le sang :}

- elle doit être réalisée en une matière évitant tout risque de thrombose,
- elle doit comporter un système de valves évitant l'hémolyse, et un tracé hydraulique évitant les turbulences ;

\section{3. en ce qui concerne la mécanique :}

- elle doit satisfaire aux exigences de la résistance, compte tenu du fait qu'il s'agit d'efforts alternés.

Cet ensemble de conditions ne laisse pas une grande marge de mancuvre, aussi la plupart des protheses sontelles assez semblables. Pour chaque cœur, l'intérieur d'une coque rigide est séparé en deux compartiments par une membrane déformable. L'un des compartiments rempli de sang est en communication avec le réseau vasculaire par deux orifices pourvus de valves, l'une d'entrée, l'autre de sortie. L'autre compartiment, rempli d'un gaz ou d'air, est en communication par un orifice sans valve avec un générateur de pulsation externe.

Ce générateur ou activateur de construction classique comprend une source de gaz sous pression, une source de gaz en dépression et un programmeur permettant de régler : la fréquence, le rapport systole/diastole, les pressions systolique et diastolique. Suivant ce schéma la source d'énergie est donc externe. Mais beaucoup de solutions ont été proposées et essayées avec une source interne directement implantable dans l'organisme. Ceci ne change en rien la structure de la prothèse ventriculaire elle-même. Jusqu'à présent aucun dispositif n'imite véritablement le cœur, la fonction volumétrique est séparée de la fonction énergétique. Aucun dispositif en particulier n'emprunte aux substrats du sang, l'énergie nécessaire ainsi que le font les cellules myocardiques.

\section{Comportement d'une prothèse en présence du réseau vasculaire}

Du point de vue purement hémodynamique qui est celui de la fonction essentielle de la prothèse, plusieurs aspects sont à considérer.

La forme de l'onde systolique obtenue doit satisfaire a certaines conditions. L'une, relative aux gradients de pression obtenus, est liée au bon fonctionnement des barorécepteurs qui interviennent dans la régulation en agissant sur la vaso-tonicité, c'est-à-dire sur les résistances périphériques pour le maintien de la pression artérielle. Leur influence normale sur la fréquence cardiaque n'est pas non plus à négliger, si par conservation du nœud sinusal on conserve les informations électriques recueillies en ce point comme élément de commande de l'activateur.

L'autre condition a trait à la propagation et réflexion des ondes de pression dans l'aorte. Le bon fonctionnement de la valve aortique dépend en effet d'une résonance de l'aorte sur l'harmonique 2 de l'onde systolique.

Dans ces conditions une systole sinusoïdale n'est pas satisfaisante. Il convient par contre d'obtenir une onde à front raide se rapprochant de la forme physiologique.

La disposition des deux cœurs en série sur le même circuit pose le problème de l'exacte adaptation de leurs đébits. Si l'égalité n'est pas obtenue, une accumulation de 


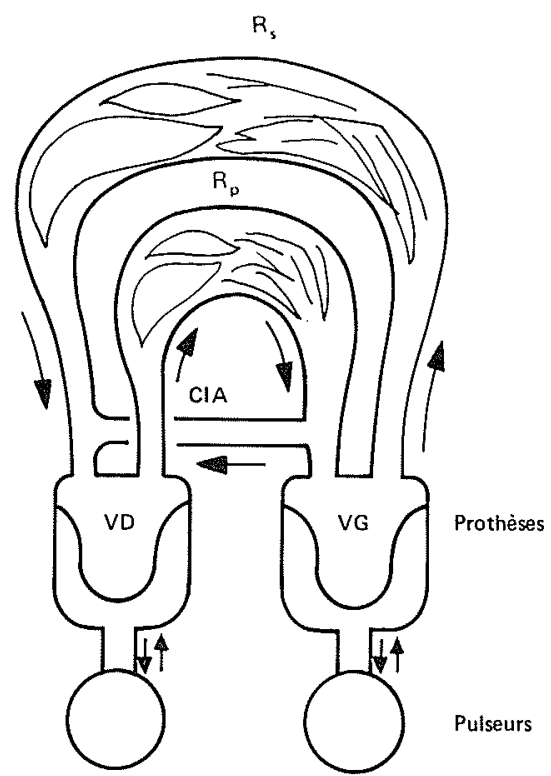

Figure 2 - Schéma d'une prothèse avec shunt auriculaire.

masse sanguine se produit sur l'une ou l'autre circulation. Une solution simple de ce problème est offerte par l'exemple d'une malformation congénitale classique : la communication intérauriculaire qui, dans certaines conditions de résistance pulmonaire, est bien tolérée.En établissant une telle communication entre les deux prothèses droite et gauche à l'étage auriculaire, on obtient une possibilité de compensation en cas de déséquilibre entre les débits des deux ventricules. Cette disposition est également avantageuse dans le cas où un déséquilibre, d'origine organique, apparaît entre les résistances des deux circulations. Normalement le rapport de ces résistances doit être égal au rapport des pressions foumies par les ventricules. On évite ainsi, en particulier par l'emploi de la communication, un accroissement de pression auriculaire gauche dont la conséquence pulmonaire est fatale.



Figure 4 - Influence de la membrane sur le débit.

Une autre solution, plus générale, consiste a adopter dans chaque prothèse une membrane déformable dont la caractéristique soit telle que le ventricule évacue à chaque systole exactement le volume qui lui est apporté par le retour veineux. Il doit exister une adaptation convenable de la déformation de la membrane à la variation de la pression du retour veineux. Or celle-ci ne varie que de quelques millimètres de mercure pour une variation de débit de l'ordre du simple au double.

En cas de déséquilibre de débit entre les deux prothèses, l'accroissement de pression veineuse sur la prothèse déficitaire doit provoquer un accroissement de volume au remplissage et par conséquent un accroissement de débit compensateur.

La correction d'un tel déficit par un réglage de la dépression diastolique à l'activateur est difficile. En effet, un excès de correction entraîne pour le débit un effet contraire à celui escompté par suite du collapsus veineux qui réduit le retour.

Le problème du déséquilibre droite-gauche apparaît également temporairement au cas où une vasodilation provoque un stockage de masse sanguine (par
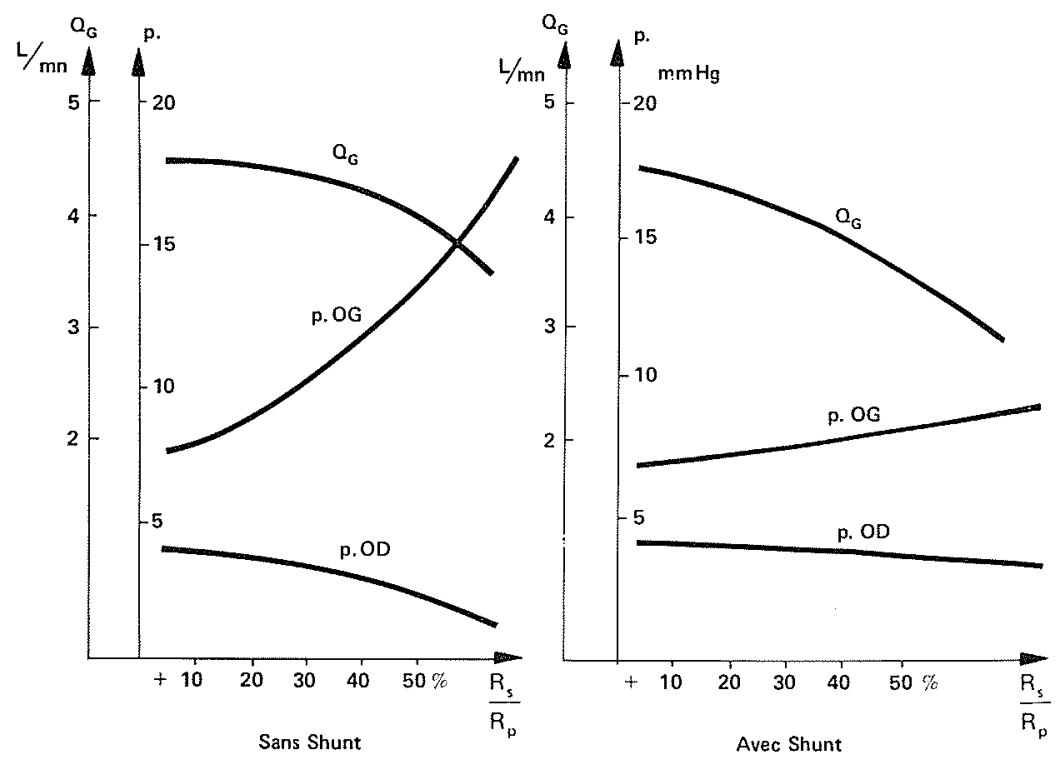

Figure 3 - Influence du shunt sur les pressions auriculaires. 
exemple dans le territoire splanchnique). La compensation par réduction de la masse sanguine pulmonaire fait apparaître le rôle important de la compliance à ce niveau.

Les difficultés qui viennent d'être évoquées mettent en lumière l'extrême sensibilité du comportement diastolique du ventricule naturel. La dilatation assurant le remplissage de ce dernier se fait, d'une part grâce à la pression veineuse et au minime apport de la contraction auriculaire, d'autre part grâce à l'élasticité propre de la paroi myocardique, élasticité d'ailleurs modifiée par la ventilation et par la viscosité des tissus. Le myocarde est un des rares muscles dont l'élongation ne soit pas facilitée par le jeu d'un muscle antagoniste.

\section{Modèle physique du système circulatoire}

La mise au point mécanique et l'étude du fonctionnement des prothèses ont incité à réaliser un modèle de système circulatoire reproduisant l'essentiel des caractéristiques hémodynamiques.

Il est bon ici de se rappeler que le but premier de la fonction circulatoire est d'assurer aux tissus les échanges métaboliques dont ils ont besoin, ce qui met au premier rang des nécessités la fréquence de renouvellement du sang capillaire.

Pour l'adulte au repos la masse sanguine effectue une révolution par minute en moyenne.

On peut alors considérer les différentes caractéristiques de fonctionnement comme formant un système bouclé.



Le débit est contrôlable par la prothèse, le volume l'est dans une certaine mesure. Résistance et compliance appartiennent étroitement au système organique. Pression et taux de renouvellement sont des conséquences de 1'ensemble. La réalisation d'un modèle devra donc porter essentiellement sur la fidèle reproduction des résistances et compliances.

Du point de vue des résistances, la plupart des modèles utilisés ne reproduisent que la valeur globale de la résistance pour un débit donné. Il est apparu qu'il était fondamental de reproduire la loi de perte de charge de ces résistances. Or il s'agit ici de pertes en régime laminaire et non pas turbulent. Le modèle que nous avons utilisé comporte des résistances constituées par des tubes fins dont le régime laminaire est assuré par le réglage de la viscosité du fluide. Le non respect de cette condition conduit à l'obtention d'ondes systoliques déformées dans leur partie initiale.

Du point de vue des compliances et des répartitions de volume il convient de distinguer les secteurs artériels et veineux des deux circulations. Dans la représentation adoptée, les quatre compartiments présentent des volumes réglables ainsi qu'une élasticité modifiables par le jeu de la pression maintenue au-dessus de la surface libre ainsi que par le volume d'air existant au-dessus de cette surface.

On a tenu compte enfin de l'étroite liaison existant dans la circulation pulmonaire entre pression-volume et résistance, un accroissement de pression et de volume

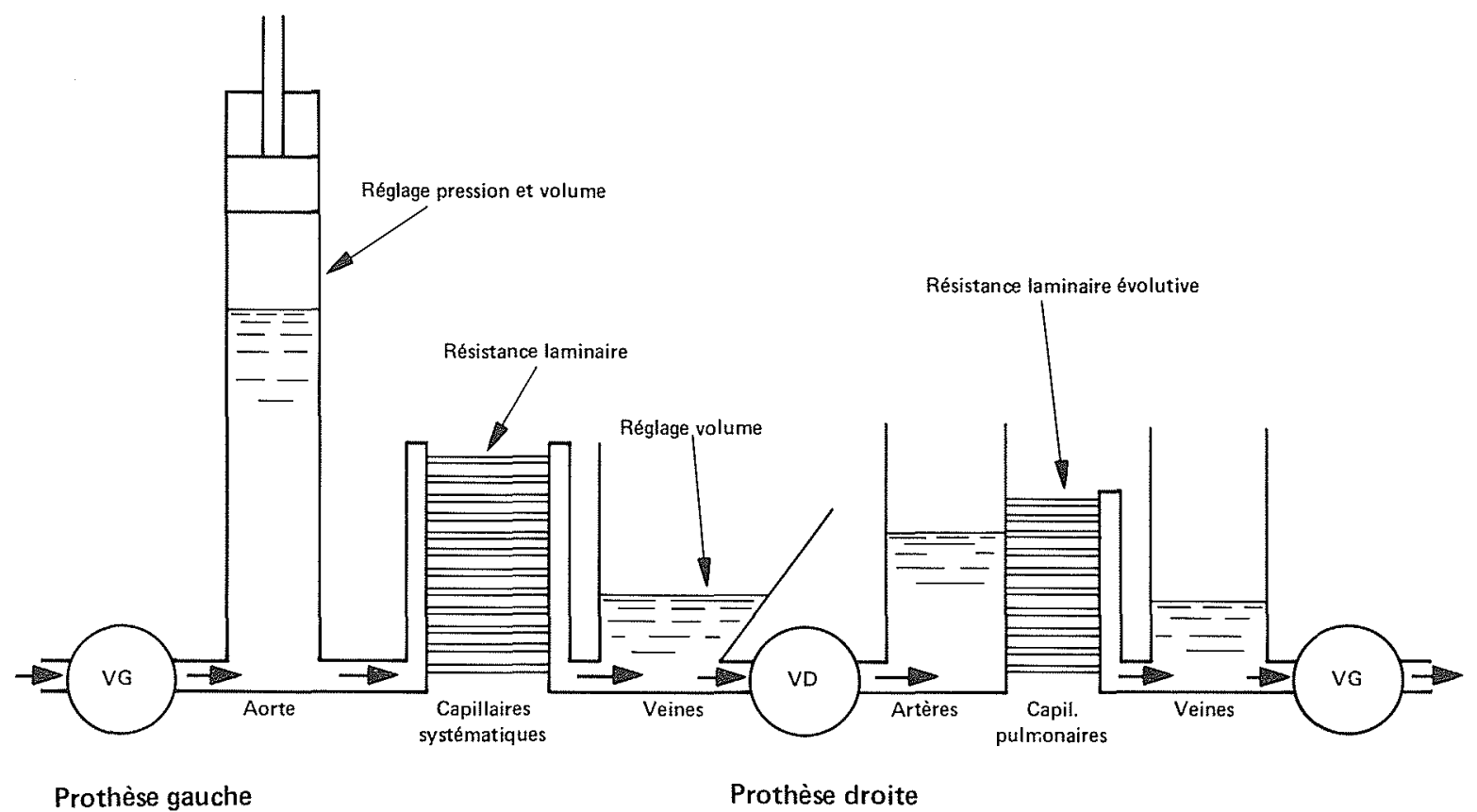

Figure 5 - Schéma du modèle circulatoire. 
entraînant une réduction de la résistance. Ceci est obtenu par un échelonnement convenable des tubes de résistance laminaire suivant la hauteur du réservoir pulmonaire.

Le modèle circulatoire répondant aux caractéristiques de résistance et compliance convenables est utilisé à plusieurs fins. Il permet tout d'abord l'analyse des comportements transitoires tels que variation de vasotonicité, variation de la masse sanguine circulante, déséquilibre droite-gauche. Dans un tel cas, certes, un modèle mathématique du système circulatoire peut être employé. On a toutefois préféré le modèle physique pour son économie de réalisation, pour sa plus grande valeur éducative, et pour la possibilité qu'il offre d'introduire directement les prothèses dans le circuit.

L'utilisation principale du modèle est bien en effet de contrôler le comportement mécanique des prothèses tant à propos d'une modification de conception que, à titre de test, avant une implantation.

Indépendamment des prothèses complètes, le modèle permet enfin l'examen de prothèses uniquement valvulaires dans des conditions très variées de fréquence et résistance.

Un tel modele est utilisé par l'équipe du professeur Marion a Lyon, pour tester les prothèses a implanter.

\section{Discussion}

Président : M.J. HINGLAIS

M. le Président remercie M. VADOT de son exposé et observe que ses orientations sont à bien des égards à l'opposé de celles que lui-même et son équie ont adoptées. C'est ainsi, précise-t-il d'abord, que "nous travaillons depuis deux ans avec des pompes centrifuges qui remplacent parfaitement les ventricules naturels, aussi bien du côté droit que du côté gauche."

D'une façon plus générale, il ne lui semble pas nécessaire de prendre en compte au niveau du cœur artificiel toutes les fonctions de régulation normalement assurées au niveau des organes en fonction de leurs besoins en oxygène: selon la loi de Starling, chaque ventricule se contente de régler son débit de sortie à la demande, c'est-à-dire en fonction du débit d'entrée qui lui arrive par la voie veineuse. Evoquant le rôle capital des "barorécepteurs" qui, par le système nerveux central, interviennent dans la physiologie, le Président indique qu'ils "agissent un peu sur la fonction ventriculaire" mais que les expérimentations montraient "que l'on pouvait parfaitement s'en passer" à ce niveau.

En définitive, conclut-il : "Le cœur est uniquement une loi de Starling. Il n'a à s'occuper de rien d'autre".

En réponse à une question de $\mathrm{M}$. COMOLET, le Président indique que, à son grand étonnement, la pompe centrifuge utilisée dans ses expériences - qui durent de 4 à 5 heures - ne provoquait pas d'hémolyse notable. Il précise à ce sujet que les pompes avaient été calculées de façon que la vitesse du sang ne dépasse nulle part $1 \mathrm{~m} / \mathrm{sec}$. alors que lorganisme présente des vitesses 2 ou 4 fois supérieures. Leur vitesse de rotation est d'environ $4000 \mathrm{tr} / \mathrm{min}$. et leur débit de 5 à 10 litres/minute. Le diamètre du rotor est de $35 \mathrm{~mm}$ et elles ont été réalisées dans un matériau extrêmement banal.

Il signale qu'aux U.S.A., BERNSTEIN a testé une petite pompe centrifuge d'assistance circulatoire et a amplement vérifié qu'elle ne provoquait qu'une faible hémolyse très supportable.

M. VADOT se montre surpris : il connaît en effet des cas où l'on est par contre surpris de constater la destruction des globules rouges alors que les vitesses de circulation sont relativement faibles.

Le Docteur SEBBAN demande au Président et à $M$. VADOT quels résultats pratiques ils ont obtenu concernant la durée de survie des animaux.

M. VADOT indique que les problèmes métaboliques sont naturellement très nombreux et très importants... mais qu'en fait bien des problèmes "économiques" - disponibilité du per- sonnel de surveillance entre autres - interviennent de façon finalement très impérative. Il mentionne également les très gros problèmes pulmonaires rencontrés sur le veau.

Le Président précise que son équipe fait plutôt de l'expérimentation "aigue", menée sous héparine avec, au bout de 4 heures, une hémorragie diffuse compromettant l'intérêt des observations. En défintivie, il pense disposer de 3 heures de "bonne période expérimentale" sur le chien.

Un auditeur ayant rappelé que certaines équipes parlaient de survie de 100 jours, le Président insiste sur la gravité de ce problème et sur la très grande modestie dont il faut faire preuve :

On a parlé de 100 heures il y a quelques années déjà. En 1970 , on disait que le cour artificiel serait en vente dans toute bonne pharmacie. Maintenant on nous parle de 100 jours, ce qui est exact pour quelques animaux. En réalité, quand on parle de coeur artificiel, il s'agit de dire à un patient : on va vous remplacer votre cœur, on vous garantit 10 ans et nous savons qu'aucune panne n'est permise.

M. ODDOU remarque cependant que si l'on examine, en fonction du temps, l'évolution de la durée de vie des cours artificiels depuis le début des recherches menées à ce sujet, on trouve une courbe relativement exponentielle.

M. BIRKUI observe que, s'agissant d'obtenir un cour artificiel à plus long terme, l'adaptation d'une pompe à débit continu - type centrifuge par exemple - paraissait satisfaisante au niveau hydrodynamique (pression, débit, vitesses...). Par contre, il demande si, au niveau de la composante hématologique qui devient fondamentale, il n'est pas préférable de recourir à un système alternatif, système choisi par la nature réservant des phases de repos, à la diastole.

M. VADOT fait remarquer que si le couur est pulsé... c'est que la nature devait assurer la continuité des tissus ... et ne pouvait pas faire autrement.

M. QUEMADA se demande si le balayage discontinu des parois vasculaires n'évite pas les dépôts qui pourraient se produire en régime continu.

Le Président évoque l'hypothèse souvent formulée selon laquelle le mécanisme de régulation impliquerait un régime à pulsations. Or au cours de ses expériences de régulation périphérique et pulmonaire, il a pu comparer des observations relevées soit en régime continu, soit en régime pulsatif: il n'a relevé aucune différence sur les régulations. 
Il estime par contre - et il est approuvé par M. VADOT que la pulsativité joue un rôle indispensable au niveau des parois vasculaires dont elle assure et entretient au moins l'élasticité.

M. BIRKUI demande si l'énergie nécessaire à l'animation des pompes à débit pulsé ou continu est d'un même ordre de grandeur et quelle part pourrait revenir, en système pulsé, aux phénomènes de résonance mécanique artérielle mis en évidence par certaines équipes.

M. VADOT pense qu'il s'agit d'un problème particulièrement difficile. Le rendement de toutes les prothèses, remarque-t-il, est assez mauvais ... mais celui du cœur naturel ne paraît pas très bon non plus. La puissance en œuvre - environ $4 \mathrm{~W}$ - est surtout consommée au niveau des résistances périphériques, artériolaires, capillaires, etc. La part de l'énergie consommée susceptible d'être influencée par le caractère pulsatif du système doit être de l'ordre de $10 \%$ seulement. Le fait que ce soit pulsé ou non ne va pas changer grand chose.

Le Président indique avoir pu mesurer des rendements meilleurs en régime continu qu'en régime pulsé. Concernant les phénomènes de mise du cœur en résonance, il n'a jamais vu un coeur s'adapter sur une fréquence qui soit justement dans les harmoniques de résonance de son aorte.

M. LEDUCQ demande si un phénomène de pompage péristaltique propre aux artères n'entrerait pas en jeu et n'expliquerait pas, par exemple, l'adaptation métabolique satisfaisante de la pompe centrifuge.

Le Président indique que ses 4 ou 5 années d'expérimentation ne lui permettent pas de réponđre.

M. VADOT ne croit pas, pour sa part, à l'existence d'effet péristaltique au niveau artériel; mais cela existerait au niveau veineux.

M. BONIS compare la "pompe" de M. VADOT à celle qu'il présentait dans sa communication sur les conduites collabables.

M. VADOT observe à ce sujet que M. BONIS n'a pas pris en compte les problèmes de tous les tissus environnants, problèmes tout à fait différents suivant que l'on considère le système pulmonaire, le système splanchnique, le système veineux,etc. 